\title{
Neuroimaging in psychiatry: from bench to bedside
}

\author{
David E. J. Linden ${ }^{1,2 *}$ and Andreas J. Fallgatter ${ }^{3}$ \\ 1 Bangor Imaging Unit, Wolfson Centre for Clinical and Cognitive Neuroscience, School of Psychology, Bangor University, Bangor, UK \\ 2 School of Medical Sciences, Bangor University, Bangor, UK \\ ${ }^{3}$ Department of Psychiatry, University of Wuerzburg, Wuerzburg, Germany
}

\section{Edited by:}

Stefano F. Cappa, Vita-Salute

San Raffaele University, Italy

Reviewed by:

Paul C. Fletcher

University of Cambridge, UK

Stefano F. Cappa, Vita-Salute

San Raffaele University, Italy

*Correspondence:

David E. J. Linden, School of

Psychology, Bangor University, Bangor

LL57 2AS, UK.

e-mail:d.linden@bangor.ac.uk

\begin{abstract}
This perspective considers the present and the future role of different neuroimaging techniques in the field of psychiatry. After identifying shortcomings of the mainly symptom-focussed diagnostic processes and treatment decisions in modern psychiatry, we suggest topics where neuroimaging methods have the potential to help. These include better understanding of the pathophysiology, improved diagnoses, assistance in therapeutic decisions and the supervision of treatment success by direct assessment of improvement in disease-related brain functions. These different questions are illustrated by examples from neuroimaging studies, with a focus on severe mental and neuropsychiatric illnesses such as schizophrenia and depression. Despite all reservations addressed in the article, we are optimistic that neuroimaging has a huge potential with regard to the above-mentioned questions. We expect that neuroimaging will play an increasing role in the future refinement of the diagnostic process and aid in the development of new therapies in the field of psychiatry.
\end{abstract}

Keywords: schizophrenia, depression, bipolar, fMRI, neurofeedback

\section{INTRODUCTION}

Psychiatric diseases are mainly diagnosed by their symptoms, with little contribution from observable signs and none from biological markers so far. This places psychiatry in a unique position compared to other medical disciplines. Although a great deal of information about disease mechanisms can be garnered from animal models, the neural correlates of the defining symptoms will ultimately have to be investigated in humans with the diverse techniques of neuroimaging. These techniques are mainly computerized tomography (CT) and magnetic resonance imaging (MRI) in different variations at the structural level, and functional magnetic resonance imaging (fMRI), positron emission tomography (PET), single photon emission computerized tomography (SPECT), near-infrared spectroscopy (NIRS), magnetoencephalography (MEG) and electroencephalography (EEG)/event-related potentials (ERP) at the functional level. The assessment of the brain pathophysiology underlying psychiatric diseases constitutes a challenge and an opportunity for the techniques of human neuroimaging because they are well-placed to unravel the structural and functional correlates of psychiatric symptoms in the brain. Moreover, they may reveal changes in information processing that precede the onset of the clinical disorder and thus provide markers of risk or prognosis.

Diagnostic uncertainty is another perennial problem of psychiatry and includes the uncertainty about the very existence of the nosological entities in use. Classical concepts of mental disorders such as schizophrenia have been variously attacked as being too broad or too narrow. Here, the hope is that neuroimaging may contribute diagnostic markers to support or refute existing categories. Finally, the monitoring of treatment effects is notoriously difficult in psychiatry. The standard approach relies heavily on changes on standardised psychopathological scales, which are based on the patients' reports on their symptoms.
Neuroimaging measures of treatment effects would thus be a very welcome, more objective, addition to the presently largely subjective outcome measures.

We will focus on studies that tried to utilise neuroimaging to understand the pathophysiology of mental disorders by identifying cognitive tasks acting as a surrogate marker (e.g., working memory in schizophrenia, theory of mind in autism), those that compared different diagnostic or at-risk groups in order to obtain diagnostic or prognostic markers, and those that integrated neuroimaging in treatment protocols.

\section{UNDERSTANDING PATHOPHYSIOLOGY}

There are essentially three ways to employ neuroimaging to help unravel the pathophysiology of mental disorders. Functional neuroimaging data can be obtained while patients experience symptoms in order to elucidate the neural circuits involved. In some cases, investigators will wait for symptoms to occur and ask patients to report them online while under investigation. Where possible and ethically admissible, symptoms can also be induced, allowing for more controlled experimental conditions. This approach may sometimes entail a reduction of ecological validity, and the successful symptom induction has to be checked with psychometric or psychophysiological measures. The discovery of primary auditory cortex activity, embedded in a wider network of language and limbic areas (Dierks et al., 1999), during auditory verbal hallucinations is an example for the former approach, the demonstration of amygdala activity in response to images of spiders in patients with arachnophobia (Dilger et al., 2003) or the provocation of panic attacks in patients with panic disorder by application of triggering substances (e.g., Zwanzger et al., 2009) for the latter. The identification of these symptom-related regions and networks may inform treatment, for example targeted interference with transcranial magnetic stimulation (TMS), as shown for example with a NIRS-guided 
repetitive TMS treatment protocol in a patient with panic disorder targeted on a frontal brain region implicated in emotion processing (Dresler et al., 2009). However, one needs to keep in mind that these pathological activations are disease-specific only in the rare cases where a disease is defined by a single symptom and this symptom does not feature in other nosological categories (as is the case for arachnophobia, but not, for example, auditory hallucinations, Linden, 2008a).

However, we have to be careful with causal inferences from observations of regional brain activity during experience of specific symptoms. Such brain activity might reflect a consequence of the symptom (e.g., fear evoked by the experience of hallucinations, or a shift of attention to the auditory domain) or compensatory mechanisms (e.g., when patients try to suppress a symptom). Moreover, even if and when one can relate the existence of the symptom directly to the observed neural activity (e.g., by disrupting it with TMS and thus suppressing the symptom), the symptom could be driven by some upstream process. The finding of fronto-temporal activation during auditory hallucinations might serve as a case in point. This activation may produce the perceptual experience of voice hearing but may not in itself be abnormal. The reason that such internally generated speech is perceived as real (rather than imagined) voices might be in a deficient monitoring mechanism with a different neural substrate.

Pathological activation patterns in mental disorders may not be confined to symptomatic periods. We assume for most mental disorders that they actually persist beyond the acutely symptomatic state, and many have a remitting-relapsing course. Some traces of the disorder should therefore also be detectable during symptom-free or remitted intervals. A second way of investigating pathophysiology with imaging is therefore through measurement of structural or functional trait markers that do not depend on a particular state. The analysis of functional connectivity during the "resting state" (which may be considered as a particular task as well) falls in this category, as does the comparison of cortical volume or thickness between patients, individuals at genetic risk, and healthy controls, or the longitudinal investigation of functional or structural brain changes across phases of a disease (Takahashi et al., 2009). The findings of structural imaging in areas such as cortical thickness or anatomical connectivity, as investigated by diffusion tensor imaging, may inform about the propensity to develop certain symptoms (Rotarska-Jagiela et al., 2009).

The third way utilises surrogate markers to obtain measures of brain activation that allow inferences about the underlying pathophysiology. Examples of frequently employed surrogate markers are working memory for schizophrenia, theory of mind for autism, emotional face processing for affective disorders and cognitive control paradigms for attention deficit/hyperactivity disorder (ADHD, e.g., Fallgatter et al., 2005). The second and third approach can be complemented by other biological techniques that probe trait markers of mental disorder. The rapidly developing field of genetic imaging (first application in Fallgatter et al., 1999), where functional or structural measures are combined with functional polymorphisms or other genetic variants, is the most prominent example of such a methods combination, but other stable individual traits, such as eye movement patterns (Klein and Ettinger, 2008) can also be applied. Genetic imaging has, for example, provided insight into the mechanism through which the dopamine system may exert influences on working memory (Herrmann et al., 2007; Diaz-Asper et al., 2008) or the serotonin system on emotion processing (Hariri et al., 2002; Hariri and Weinberger, 2003). It has also been suggested that some of the structural changes seen in groups of patients with mental disorders are mediated by functional variants in growth factors, for example Brain Derived Neurotrophic Factor (BDNF; Frodl et al., 2007). This approach can proceed in two directions, starting from a gene that has been associated with a particular disorder in a linkage or association study (for example, dysbindin (Fallgatter et al., 2006) or ZNF804A (O'Donovan et al., 2008) with schizophrenia), or starting from a gene that impacts on a biochemical mechanism implicated in the pathophysiology of mental disorders (Canli and Lesch, 2007). If starting from a gene with a known association with a disorder but an unknown function, this approach can provide the first glimpses into its pathophysiological role (Esslinger et al., 2009). Conversely, starting from a biochemical mechanism, this approach can help identify intermediate phenotypes, such as heightened amygdala response or impaired working memory, which can then be further investigated for their association with mental disorders. However, in both cases the diagnostic specificity will be low, because there are commonly many more participants with the at-risk polymorphism who do not suffer from the disorder than those who do, considering the relatively low prevalence rates and low relative risk afforded by these polymorphisms.

Finally, neuroimaging measures may also be considered as intermediate phenotypes or endophenotypes which are more closely correlated with the fundamental (e.g., genetic) abnormalities of psychiatric disorders than the behaviour and the clinical symptoms themselves (see Figure 1).

Such neuroimaging endophenotypes may be applied as potential markers for the occurrence of hidden cognitive processes and provide evidence in favour of, or against, particular cognitive neuropsychiatric models of symptoms. One could also envisage that such endophenotypic measures may help refine the distinction between symptoms that are very similar at the phenomenological level. For example, it is often difficult in clinical practice to differentiate delusions of schizophrenia from those of acute mania. If neuroimaging gave us specific patterns of regional/neurochemical activation during these states, it might help us improve the current diagnostic classification systems and provide a more principled basis for the taxonomy of mental disorders.

\section{IMPROVING DIAGNOSIS}

Current diagnostic systems in psychiatry are mainly based on patients' self-reports of symptoms and to some extent also on observed behaviour, and on their course over time. For example, the criteria of the World Health Organisation's International Classification of Disease, 10th edition (ICD-10) for schizophrenia stipulate that at least one of the symptoms listed in Table $\mathbf{1}$ is present for 1 month. It becomes immediately clear that two patients can be diagnosed with schizophrenia without sharing any clinical features. Conversely they may have more in common with patients who fall into other diagnostic categories. For example a patient with symptoms from groups b and c may overlap more with a patient with bipolar affective disorder (BD) who is currently in a manic 


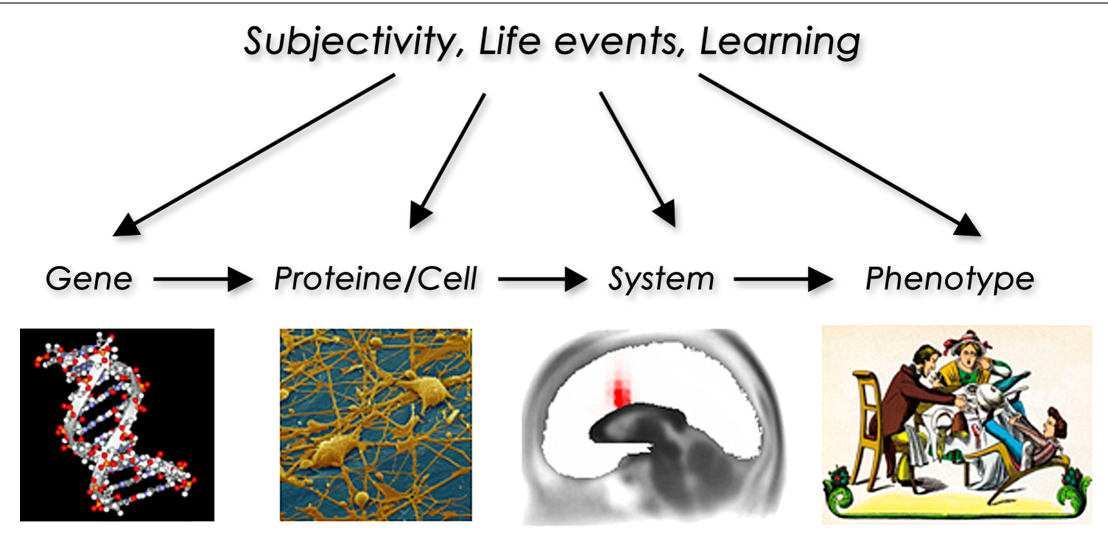

FIGURE 1 |The endophenotype concept illustrated using the example of attention deficit/hyperactivity disorder showing the way from genetic information to the levels of cell and systems function to a clinical phenotype. Additional influences by other factors may occur at every level.

Table 1 | Core symptoms of schizophrenia according to the ICD-10 (quoted verbatim from http://www.who.int/classifications/icd/en/ bluebook.pdf, accessed on 8 July 2009). One symptom of this list, clearly present over at least one month, is sufficient for the diagnosis to be made.

a) Thought echo, thought insertion or withdrawal, and thought broadcasting.

b) Delusions of control, influence, or passivity, clearly referred to body or limb movements or specific thoughts, actions, or sensations; delusional perception.

c) Hallucinatory voices giving a running commentary on the patient's behaviour, or discussing the patient among themselves, or other types of hallucinatory voices coming from some part of the body.

d) Persistent delusions of other kinds that are culturally inappropriate and completely impossible, such as religious or political identity, or superhuman powers and abilities (e.g., being able to control the weather, or being in communication with aliens from another world).

episode with psychotic features than with another schizophrenia patient who exclusively reports symptoms from group a. Against such a critique of the diagnostic validity of concepts such as "schizophrenia" or "mania", which is based on the cross-sectional overlap of symptoms, it can be maintained that commonalities and differences between the current diagnostic categories often become more apparent over time, in the natural history of symptoms and patterns of behaviour. However, even psychiatrists who believe in the usefulness of the current diagnostic concepts because of their predictive (common courses and treatment responses that differentiate, for example, bipolar disorder from schizophrenia) or genetic validity accept that additional diagnostic markers, derived from cognitive neuroscience or biology, would be of great importance.

Biological markers might also aid in the classification of disorders when the course is not yet known, for example to differentiate between unipolar depression and BD after a first episode of depression (Phillips and Vieta, 2007). Finally, there is considerable hope that they might help predict the outbreak of a clinical disorder in people at risk who are identified based on genetic (e.g., children of patients with BD, Phillips et al., 2008), clinical (e.g., mild cognitive impairment, Prvulovic et al., 2005) or a combination of these criteria (e.g., the schizophrenia prodrome, Bender et al., 2007;
Koutsouleris et al., 2009). In the absence of preventative treatments of affective disorders, Alzheimer's disease or schizophrenia, the value of such information may be questioned. However, advocates of this line of research would argue that any investigation into the preventative properties of currently used (or new) pharmacological agents would need improved criteria for early detection and that these will probably have to include biological markers.

What, then, can neuroimaging contribute to the diagnosis of mental disorders? The main contribution of structural imaging in clinical practice is to exclude other causes that may lead to similar symptoms, such as space occupying lesions, cerebrovascular or inflammatory disease, although the cost effectiveness of screening programmes has been debated (Albon et al., 2008). In the realm of severe mental illness, it is still not possible to identify patients with imaging findings alone, or to differentiate between different disorders, as discussed below.

\section{SCHIZOPHRENIA}

One of the first discoveries that were made with neuroimaging techniques in any mental disorder was the enlargement of the lateral and third ventricles in schizophrenia. This anatomical characteristic was specific to patients with schizophrenia compared to their siblings, occurred at the onset of the disease and was associated with poorer treatment response. A recent study confirmed the specificity of ventricular enlargement compared to affective psychosis (McDonald et al., 2006). Patients with schizophrenia as a group also show loss of local brain volume in numerous areas, including the language and auditory areas of the temporal lobe, the attention and visuomotor integration areas of the parietal lobe, the memory and executive areas of the frontal lobe, and parts of the limbic system and the basal ganglia. Such volume loss has been reported for schizophrenia patients already during adolescence and was found to progress at a rate of up to $4 \%$ per year (Toga et al., 2006). Furthermore, even patients with prodromal schizophrenia show limbic alterations (Pantelis et al., 2003). However, at post-mortem there is often no difference in overall brain volume or that of structures implicated in the pathogenesis of schizophrenia, e.g., the frontal lobes (Highley et al., 2001), between patients and age- and intelligencematched controls. Thus, brain degeneration does not progress at 
that pace across the lifetime in most schizophrenia patients. In sum, although several structural measures, including reduced wholebrain volume, enlarged ventricles, reduced hippocampal volume and reduced asymmetry of the temporal plane, have been associated with schizophrenia (Kwon et al., 1999; McCarley et al., 1999; Gur et al., 2007), the overlap between patients and healthy controls is too large for them to attain the specificity and sensitivity required for a diagnostic marker.

\section{AFFECTIVE DISORDERS}

Affective disturbance is perhaps the most frequent and most often neglected psychiatric manifestation of neurological disease, and thus of great clinical relevance beyond the field of classical mental disorders. Affective disturbance secondary to neurological disorders could also potentially provide clues as to the brain areas whose dysfunction leads to depression. Depressive symptoms occur frequently (in up to a third of cases) in stroke, traumatic brain injury or brain tumour, particularly those affecting the frontal lobe and basal ganglia. Although such findings could potentially provide a lead to the role of these areas in maintaining emotional balance, caution is warranted for a number of reasons. The depressive syndrome is inherently heterogeneous, and focal lesions may predominantly produce symptoms that can be part of this syndrome, but also occur during a range of other mental disorders, for example psychomotor slowing and apathy after frontal lesions. Moreover, multiple sclerosis, a disorder with a diffuse lesion pattern and predominantly white matter pathology, can also present with depression early on. The same is true for system illnesses affecting the brain such as acquired immunodeficiency syndrome, thyroid disease or even heart failure (e.g., Angermann et al., 2007). Thus, a wide range of different lesion patterns affecting both gray and white matter can produce depressive symptoms, calling any straightforward structure-function relationship into question. Finally, the interpretation of affective changes in severely disabling neurological disorders is complicated by the occurrence of adjustment disorders with depressive symptoms, which are probably linked more directly with the dysfunctional coping mechanisms of the patient than with the function of the lesioned brain area.

There is thus very little basis to expect that evidence from neuropsychiatric cases of depression will highlight the brain areas that are involved in classical psychiatric depression as well. However, there are a number of structural imaging findings from patients with unipolar or bipolar depression, which are worth considering. Evidence for ventricular enlargement is weaker in affective disorders than in schizophrenia, and a considerable number of studies found no difference between patients and controls. Elderly patients with depression have so far been the only group were a sizeable majority of studies have reported enlarged lateral ventricles (Beyer and Krishnan, 2002), and most studies in BD converge to suggest enlarged third ventricles. Strasser et al. (2005) found ventricular enlargement in schizophrenia and BD patients, but only those with the psychotic subtype. Volume losses in medial thalamic or hypothalamic areas that form the walls of the third ventricle have been suggested to underlie this enlargement. A possible implication of the hypothalamus would be of particular interest because of the prominent somatic and autonomic symptoms, for example the disturbed circadian clock, present during both depressed and manic episodes. However, the recent study by McDonald et al. (2006), which reported enlargement of lateral and third ventricles in schizophrenia, found no differences between psychotic BD patients and controls. The heterogeneity of the findings across studies and the effect of age suggest that ventricle size, if altered in affective disorders at all, would be a state rather than a trait marker. This view would be supported by the observation that relatives of patients with familial schizophrenia, but not those of patients with familial BD, also showed increased ventricles (McDonald et al., 2006). Similar findings of lack of genetic association in BD have recently been reported for corpus callosum size (Walterfang et al., 2009).

Findings are also inconsistent across studies for most cortical areas. Yet, some confined areas have been suggested by several studies to be reduced. The medial temporal lobe, particularly the hippocampus and the amygdala, has been specifically targeted by several volumetric studies because of the link between their presumed function in emotion regulation and associative memory formation and the clinical symptoms of depression. Furthermore, an attractive aetiological model, largely based on animal studies, has implicated hippocampal damage due to stress in the brain processes of depression. However, findings from structural imaging are inconsistent, with volume reductions, increases and no changes all being reported (Beyer and Krishnan, 2002). A recent report also suggests that hippocampi are smaller already during adolescence in never medicated patients with early onset familial depression (MacMaster et al., 2008). Yet, small hippocampi have also been observed in traumatised individuals without depressive symptoms (Karl et al., 2006), and this finding may thus be a non-specific consequence of stress rather than a specific feature of the pathophysiology of depression. In the basal ganglia, several studies reported a reduction of the volume of the striatum (caudate nucleus and putamen) mainly in elderly depressed patients. A link between basal ganglia pathology and depression is also suggested by the high occurrence of depression in Parkinson's disease (up to 50\%), but the apparent age effect would suggest that this may not be a primary pathology of psychiatric depression but an effect of the disease and/or medication, or a state marker of depression in the elderly.

Research in the 1980s suggested that a subgroup of patients with depression is suffering from increased secretion of cortisol and other signs of dysregulation of the hypothalamic-pituitary-adrenal (HPA) axis. This line of research has recently attracted renewed interested because of the HPA-stress model of depression, which will be discussed below. Structural imaging lent some support to changes in the HPA in depression. Both the adrenal gland and the pituitary were found to be enlarged in patients with depression, but it is as yet unknown whether this would normalise again after successful treatment or remission. In any case, as with most changes in the HPA axis, it will be difficult if not impossible to determine whether they are aetiological factors of depression or correlates of the stress resulting from depression, unless they are found in subjects with genetic risk but no clinical symptoms.

\section{TREATMENT EFFECTS}

Although the best outcome measures of a psychiatric or psychological intervention will always be at the level of the clinical phenotype - for example, improvement of mood in a case of 
depression - these are sometimes difficult to define or obtain, especially for not primarily symptom-focussed techniques. This difficulty and the unreliability of self-report measures have triggered a quest for biological or physiological markers of treatment responses. Such markers could be obtained by psychophysiology (e.g., galvanic skin response to emotional pictures), biochemistry (e.g., basal cortisol levels), neurophysiology (e.g., changes in the P100 or P300 components of the visual event-related potential, Fallgatter et al., 2003; Haenschel et al., 2007), receptor mapping (e.g., changes in striatal dopamine receptor occupancy, Hiemke, 2008), or, functional neuroimaging with fMRI, SPECT, PET, or NIRS. The attraction of functional neuroimaging is that it provides a means of assessing metabolism or activation of individual brain regions, which confers an advantage compared to more global (biochemical or psychophysiological) measures where treatment effects are expected to operate through modifications of specific pathophysiological nodes. For example, if spider phobia is indeed caused by hyperactivation of the limbic system (Dilger et al., 2003), a successful intervention should lead to a reduced response of implicated areas to the triggering stimulus.

Treatment monitoring with functional neuroimaging has had particularly fascinating applications in the field of psychological interventions. One interesting aspect of this approach was that it had to overcome longstanding mutual reservations on the part of psychotherapists and neuroscientists. We can now safely say that there has been a paradigm shift over the past 10 years to an integrated view of psychological and neurobiological models of mental illness and its treatment. We have recently reviewed the neuroimaging studies that investigated psychotherapy effects or compared psycho- and pharmacotherapy effects for anxiety disorders and depression elsewhere (Linden, 2006, 2008b). Several studies of cognitive behavioural therapy for specific or social phobia indeed found decreased activity in limbic structures (Furmark et al., 2002; Paquette et al., 2003; Straube et al., 2006) after successful treatment. The relationship between psycho- and pharmacotherapy can be studied best in disorders where both approaches are equally potent and commonly used. For example, in the study of social phobia a similar reduction in medial temporal lobe activity during preparation of a public speech was observed in the CBT and antidepressant treated groups (Furmark et al., 2002).

What contribution can functional neuroimaging then make to the treatment of mental disorders? We suggest three lines of investigation and potentially practical implementation (Linden, 2008c):

(a) Understand the mechanisms of neural plasticity that can be utilised in psychological and pharmacological interventions.

(b) Compare neural changes during different types of intervention (psycho- vs. pharmacotherapy and between different psychological interventions). It may ultimately become possible to identify likely responders to different types of therapy by their baseline patterns of neural activation, allowing for an individualized therapy. A word of caution is needed here because this aim has not been fulfilled in over a decade of functional imaging of pharmacotherapy, and refined psychopathological criteria may be at least as promising. (c) Provide information about the pathophysiological nodes that may become the targets for physical (TMS, deep brain stimulation: DBS) or neurofeedback intervention.

\section{DEVELOPING NEW THERAPIES}

Functional imaging results have influenced or even motivated various physical treatment approaches. The finding of auditory cortex activity during hallucinations has already led to partly successful attempts at attenuating hallucinations with TMS over the temporal lobe (Hoffman et al., 2003; Jandl et al., 2006). In affective disorders, attention has focused on the subgenual area of the medial frontal lobe, where both volume and metabolism were found to be reduced (Drevets et al., 1997). However, when metabolic activity was corrected for the partial volume effect, it appears to be higher in depressed patients compared to controls and revert to normal after effective treatment. This area has been the target of a recent DBS study with treatment refractory depressed patients (Lozano et al., 2008), but its clinical effects still await confirmation by a controlled trial. The recent finding of contradictory treatment effects in the same brain area (Kennedy et al., 2007) also underlines that one has to be careful in associating a complex syndrome with a single putative area of hyperactivity.

Another interesting development driven by the advances of fMRI is the self-regulation of brain activity through neurofeedback. This technique consists in training participants to influence the neural signal from a target area while they receive online information about the amplitude of this signal (Weiskopf et al., 2003; deCharms, 2007; Johnston et al., 2010). The rationale for its therapeutic application can perhaps be based on the analogy with hypnosis. Hypnotic suggestions can attenuate somatosensory cortex and amygdala activity as well as pain perception (Röder et al., 2007). It should therefore be possible in principle for participants to produce similar changes themselves, for example by specific imagery strategies. FMRI-neurofeedback, targeting the anterior cingulated cortex, has indeed shown some preliminary success in chronic pain (deCharms et al., 2005). However, any application in complex psychiatric disorders probably would have to integrate neurofeedback in a comprehensive biopsychosocial intervention package, as one would with classical biofeedback or relaxation techniques. However, if we believe that dysfunctional activity in specific areas or networks of the brain makes a causal contribution to mental illness, the ability to self-regulate these areas should ultimately have a beneficial effect on a person's mental health.

\section{CONCLUSION}

Functional and structural neuroimaging are key techniques of modern brain research and play a major role in the quest for biological markers of mental disorders. Although they have already contributed greatly to our understanding of the pathophysiology of mental disorders and their associated symptoms, clinical applications in diagnosis or treatment monitoring are still sparse. However, they may become more widespread once more information about the reliability and diagnostic specificity of neuroimaging changes becomes available. Neuroimaging is also likely to assume increasing importance as a tool to monitor and compare the brain effects of different psychiatric and psychological treatments, and eventually aid in the development of new interventions. 


\section{REFERENCES}

Albon, E., Tsourapas, A., Frew, E., Davenport, C., Oyebode, F., Bayliss, S., Arvanitis, T., and Meads, C. (2008). Structural neuroimaging in psychosis: a systematic review and economic evaluation. Health Technol. Assess. 12, iii-iv, ix-163.

Angermann, C. E., Gelbrich, G., Störk, S., Fallgatter, A. J., Deckert, J., Faller, H., and Ertl, G. (2007). Rationale and design of a randomised, controlled, multicenter trial investigating the effects of selective serotonin re-uptake inhibition on Morbidity, mortality and mood in Depressed Heart Failure patients (MOOD-HF). Eur. J. Heart Fail. 9, 1212-1222.

Bender, S., Weisbrod, M., and Resch, F. (2007). Which perspectives can endophenotypes and biological markers offer in the early recognition of schizophrenia? J. Neural Transm. 114, 1199-1215.

Beyer, J., and Krishnan, K. (2002). Volumetric brain imaging findings in mood disorders. Bipolar Disord. $4,89-104$.

Canli, T., and Lesch, K. (2007). Long story short: the serotonin transporter in emotion regulation and social cognition. Nat. Neurosci. 10, 1103-1109.

deCharms, R. (2007). Reading and controlling human brain activation using real-time functional magnetic resonance imaging. Trends Cogn. Sci. $11,473-481$.

deCharms, R., Maeda, F., Glover, G., Ludlow, D., Pauly, J., Soneji, D., Gabrieli, J., and Mackey, S. (2005). Control over brain activation and pain learned by using real-time functional MRI. Proc. Natl. Acad. Sci. U.S.A. 102, 18626-18631.

Diaz-Asper,C.,Goldberg, T., Kolachana, B., Straub,R.,Egan, M., and Weinberger, D. (2008). Genetic variation in catecholO-methyltransferase: effects on working memory in schizophrenic patients, their siblings, and healthy controls. Biol. Psychiatry 63, 72-79.

Dierks, T., Linden, D., Jandl, M., Formi sano, E., Goebel, R., Lanfermann, H., and Singer, W. (1999). Activation of Heschl's gyrus during auditory hallucinations. Neuron 22, 615-621.

Dilger, S., Straube, T., Mentzel, H., Fitzek, C., Reichenbach, J., Hecht, H., Krieschel, S., Gutberlet, I., and Miltner, W. (2003). Brain activation to phobia-related pictures in spider phobic humans: an event-related functional magnetic resonance imaging study. Neurosci. Lett. 348, 29-32.

Dresler, T., Ehlis, A. C., Plichta, M. M., Richter, M. M., Jabs, B., Lesch, K. P., and Fallgatter, A. J. (2009). Panic disorder and a possible treatment approach by means of high-frequency rTMS: A case report. World J. Biol. Psychiatry 24, 1-7.

Drevets, W., Price, J., Simpson, J. J., Todd, R., Reich, T., Vannier, M., and Raichle, M. (1997). Subgenual prefrontal cortex abnormalities in mood disorders. Nature 386, 824-827.

Esslinger, C., Walter, H., Kirsch, P., Erk, S., Schnell, K., Arnold, C., Haddad, L., Mier, D., Opitz von Boberfeld, C., Raab, K., Witt, S., Rietschel, M., Cichon, S., and Meyer-Lindenberg, A. (2009). Neural mechanisms of a genome-wide supported psychosis variant. Science 324, 605.

Fallgatter, A. J., Bartsch, A. J., Zielasek, J., and Herrmann, M. J. (2003). Brainelectrical dysfunction of the anterior cingulate in schizophrenic patients. Psychiatry Res. Neuroimaging $124,37-48$.

Fallgatter, A. J., Ehlis, A. C., Rösler, M., Strik, W. K., Blocher, D., and Herrmann, M. J. (2005). Diminished prefrontal brain function in adults with attention deficit hyperactivity disorder (ADHD) related psychopathology in childhood. Psychiatry Res. Neuroimaging 138, 157-169.

Fallgatter,A.J.,Herrmann,M.J.,Hohoff, C., Ehlis, A. C., Jarczok, T. A., Freitag, C. M., and Deckert, J. (2006). DTNBP1 (Dysbindin) gene variants modulate prefrontal brain function in healthy individuals. Neuropsychopharmacology 31, 2002-2010.

Fallgatter, A. J., Jatzke, S., Bartsch, A. J., Hamelbeck, B., and Lesch, K. P. (1999). Serotonin transporter promoter polymorphism influences topography of inhibitory motor control. Int. J. Neuropsychopharmacology 2, 115-120.

Frodl, T., Schüle, C., Schmitt, G., Born, C., Baghai, T., Zill, P., Bottlender, R., Rupprecht, R., Bondy, B., Reiser, M., Möller, H., and Meisenzahl, E. (2007). Association of the brain-derived neurotrophic factor Val66Met polymorphism with reduced hippocampal volumes in major depression. Arch. Gen. Psychiatry 64, 410-416.

Furmark, T., Tillfors, M., Marteinsdottir,I., Fischer, H., Pissiota, A., Långström, B., and Fredrikson, M. (2002). Common changes in cerebral blood flow in patients with social phobia treated with citalopram or cognitive-behavioral therapy. Arch. Gen. Psychiatry $59,425-433$.

Gur, R., Keshavan, M., and Lawrie, S. (2007).Deconstructing psychosis with human brain imaging. Schizophr. Bull. 33, 921-931.

Haenschel, C., Bittner, R., Haertling, F., Rotarska-Jagiela, A., Maurer, K., Singer, W., and Linden, D. (2007).
Contribution of impaired early-stage visual processing to working memory dysfunction in adolescents with schizophrenia: a study with eventrelated potentials and functional magnetic resonance imaging. Arch Gen. Psychiatry 64, 1229-1240.

Hariri, A., Mattay, V., Tessitore, A., Kolachana, B., Fera, F., Goldman, D., Egan, M., and Weinberger, D. (2002). Serotonin transporter genetic variation and the response of the human amygdala. Science 297, 400-403.

Hariri, A., and Weinberger, D. (2003) Functional neuroimaging of genetic variation in serotonergic neurotransmission. Genes Brain Behav. 2, 341-349.

Herrmann, M. J., Walter, A., Schreppel, T. Ehlis, A. C., Pauli, P., Lesch, K. P., and Fallgatter, A. J. (2007). D4 receptor gene variation modulates activation of prefrontal cortex during working memory. Eur. J. Neurosci. 26 2713-2718.

Hiemke, C. (2008). Therapeutic drug monitoring in neuropsychopharmacology: does it hold its promises? Eur. Arch. Psychiatry Clin. Neurosci. 258(Suppl. 1), 21-27.

Highley, J., Walker, M., Esiri, M., McDonald, B., Harrison, P., and Crow, T. (2001). Schizophrenia and the frontal lobes: post-mortem stereological study of tissue volume. Br. J. Psychiatry 178, 337-343.

Hoffman, R., Hawkins, K., Gueorguieva, R., Boutros, N., Rachid, F., Carroll, K., and Krystal, J. (2003). Transcranial magnetic stimulation of left temporoparietal cortex and medication resistant auditory hallucinations. Arch Gen. Psychiatry 60, 49-56.

Jandl, M., Steyer, J., Weber, M., Linden, D., Rothmeier, J., Maurer, K., and Kaschka, W. (2006). Treating auditory hallucinations by transcranial magnetic stimulation: a randomized controlled cross-over trial. Neuropsychobiology 53, 63-69.

Johnston, S. J., Boehm, S. G., Healy, D., Goebel, R., and Linden, D. E. (2010) Neurofeedback: a promising tool for the self-regulation of emotion networks. Neuroimage 49, 1066-1072. [Epub ahead of print].

Karl, A., Schaefer, M., Malta, L., Dörfel, D. Rohleder, N., and Werner, A. (2006) A meta-analysis of structural brain abnormalities in PTSD. Neurosci. Biobehav. Rev. 30, 1004-1031.

Kennedy, S., Konarski, J., Segal, Z., Lau, M., Bieling, P., McIntyre, R., and Mayberg, H. (2007). Differences in brain glucose metabolism between responders to CBT and venlafaxine in a 16 -week randomized controlled trial. Am. J. Psychiatry 164, 778-788.
Klein, C., and Ettinger, U. (2008). A hundred years of eye movement research in psychiatry. Brain Cogn. $68,215-218$.

Koutsouleris, N., Meisenzahl, E., Davatzikos, C., Bottlender, R., Frodl, T., Scheuerecker, J., Schmitt, G., Zetzsche, T., Decker, P., Reiser, M., Möller, H., and Gaser, C. (2009). Use of neuroanatomical pattern classification to identify subjects in at-risk mental states of psychosis and predict disease transition. Arch. Gen. Psychiatry $66,700-712$.

Kwon, J., McCarley, R., Hirayasu, Y., Anderson, J., Fischer, I., Kikinis, R., Jolesz, F., and Shenton, M. (1999). Left planum temporale volume reduction in schizophrenia. Arch. Gen. Psychiatry $56,142-148$.

Linden, D. (2006). How psychotherapy changes the brain--the contribution of functional neuroimaging. Mol. Psychiatry 11, 528-538.

Linden, D. (2008a). Auditory hallucinations and functional imaging). Fortschr. Neurol. Psychiatr. 76(Suppl. 1), S33-S39.

Linden, D. (2008b). Brain imaging and psychotherapy: methodological considerations and practical implications. Eur. Arch. Psychiatry Clin. Neurosci. 258(Suppl. 5), 71-75.

Linden, D. (2008c). Changing the mind, changing the brain. NeuroPsychoanalysis 10, 26-28.

Lozano, A., Mayberg, H., Giacobbe, P., Hamani, C., Craddock, R., and Kennedy, S. (2008). Subcallosal cingulate gyrus deep brain stimulation for treatment-resistant depression. Biol. Psychiatry 64, 461-467.

MacMaster, F., Mirza, Y., Szeszko, P., Kmiecik, L., Easter, P., Taormina, S., Lynch, M., Rose, M., Moore, G., and Rosenberg, D. (2008). Amygdala and hippocampal volumes in familial early onset major depressive disorder. Biol. Psychiatry 63, 385-390.

McCarley, R., Wible, C., Frumin, M., Hirayasu, Y., Levitt, J., Fischer, I., and Shenton, M. (1999). MRI anatomy of schizophrenia. Biol. Psychiatry 45, 1099-1119.

McDonald, C., Marshall, N., Sham, P. Bullmore, E., Schulze, K., Chapple, B., Bramon, E., Filbey, F., Quraishi, S., Walshe, M., and Murray, R. (2006). Regional brain morphometry in patients with schizophrenia or bipolar disorder and their unaffected relatives. Am. J. Psychiatry 163, 478-487.

O'Donovan, M.,Craddock, N.,Norton, N., Williams, H., Peirce, T., Moskvina, V., Nikolov, I., Hamshere, M., Carroll, L., Georgieva, L., Dwyer, S., Holmans, P., Marchini, J., Spencer, C., Howie, B., Leung, H., Hartmann, A., Möller, H., 
Morris, D., Shi,Y., Feng, G., Hoffmann, P., Propping, P., Vasilescu, C., Maier W., Rietschel, M., Zammit, S., Schumacher, J., Quinn, E., Schulze, T., Williams, N., Giegling, I., Iwata, N., Ikeda, M., Darvasi, A., Shifman, S., He, L., Duan, J., Sanders, A., Levinson, D., Gejman, P., Cichon, S., Nöthen, M., Gill, M., Corvin, A., Rujescu, D., Kirov, G., Owen, M., Buccola, N., Mowry, B., Freedman, R., Amin, F., Black, D., Silverman, J., Byerley, W., and Cloninger, C. (2008). Identification of loci associated with schizophrenia by genome-wide association and followup. Nat. Genet. 40, 1053-1055.

Pantelis, C., Velakoulis, D., McGorry, P., Wood, S., Suckling, J., Phillips, L., Yung, A., Bullmore, E., Brewer, W., Soulsby, B., Desmond, P., and McGuire, P. (2003). Neuroanatomical abnormalities before and after onset of psychosis: a cross-sectional and longitudinal MRI comparison. Lancet 361, 281-288.

Paquette, V., Lévesque, J., Mensour, B., Leroux, J., Beaudoin, G., Bourgouin, P., and Beauregard, M. (2003). "Change the mind and you change the brain": effects of cognitive-behavioral therapy on the neural correlates of spider phobia. Neuroimage 18, 401-409.

Phillips, M., Ladouceur, C., and Drevets, W. (2008). A neural model of voluntary and automatic emotion regulation: implications for understanding the pathophysiology and neurodevelopment of bipolar disorder. Mol. Psychiatry 13, 829-857.

Phillips, M., and Vieta, E. (2007). Identifying functional neuroimaging biomarkers of bipolar disorder: toward DSM-V. Schizophr. Bull. 33, 893-904.

Prvulovic, D., Van de Ven, V., Sack, A., Maurer, K., and Linden, D. E. (2005). Functional activation imaging in aging and dementia. Psychiatry Res $140,97-113$.

Röder, C., Michal, M., Overbeck, G., van de Ven, V., and Linden, D. (2007). Pain response in depersonalization: a functional imaging study using hypnosis in healthy subjects. Psychother. Psychosom. 76, 115-121.

Rotarska-Jagiela, A., Oertel-Knoechel, V., Demartino, F., van de Ven, V., Formisano, E., Roebroeck, A., Rami, A., Schoenmeyer, R., Haenschel, C., Hendler, T., Maurer, K., Vogeley, K., and Linden, D. E. (2009). Anatomical brain connectivity and positive symptoms of schizophrenia: A diffusion tensor imaging study. Psychiatry Res. 174, 9-16. [Epub ahead of print].

Strasser, H., Lilyestrom, J., Ashby, E., Honeycutt, N., Schretlen, D., Pulver, A. Hopkins, R., Depaulo, J., Potash, J., Schweizer, B., Yates, K., Kurian, E., Barta, P., and Pearlson, G. (2005). Hippocampal and ventricular volumes in psychotic and nonpsychotic bipolar patients compared with schizophrenia patients and community control subjects: a pilot study. Biol. Psychiatry 57, 633-639.

Straube, T., Glauer, M., Dilger, S., Mentze, H., and Miltner, W. (2006) Effects of cognitive-behavioral therapy on brain activation in specific phobia Neuroimage 29, 125-135.

Takahashi, T., Yücel, M., Lorenzetti, V., Tanino, R., Whittle, S., Suzuki, M. Walterfang, M., Pantelis, C., and Allen, N. (2009). Volumetric MRI study of the insular cortex in individuals with current and past major depression. J. Affect.Disord. Electronic publication 17 Sept.

Toga, A., Thompson, P., and Sowell, E. (2006). Mapping brain maturation. Trends Neurosci. 29, 148-159.

Walterfang, M., Wood, A., Barton, S. Velakoulis, D., Chen, J., Reutens, D. Kempton, M., Haldane, M. Pantelis, C., and Frangou, S. (2009). Corpus callosum size and shape alterations in individuals with bipolar disorder and their first-degree relatives. Prog. Neuropsychopharmacol. Biol. Psychiatry 33, 1050-1057.

Weiskopf, N., Veit, R., Erb,M.,Mathiak, K., Grodd, W., Goebel, R., and Birbaumer, N. (2003). Physiological self-regulation of regional brain activity using real-time functional magnetic resonance imaging (fMRI): methodology and exemplary data. Neuroimage 19, 577-586.

Zwanzger,P.,Eser, D., Romeo,E., di Michele, F., Baghai, T. C., Pasini, A., Padberg, F., and Rupprecht, R. (2009). Changes in CCK-4 induced panic after treatment with the GABA-reuptake inhibitor tiagabine are associated with an increase in 3alpha, 5alpha-tetrahydrodeoxycorticosterone concentrations. Psychoneur oendocrinology, 116, 767-775.

Conflict of Interest Statement: The authors declare that the research was conducted in the absence of any commercial or financial relationships that could be construed as a potential conflict of interest.

Received: 23 July 2009; paper pending published: 03 October 2009; accepted: 02 November 2009; published online: 23 December 2009

Citation: Linden DEJ and Fallgatter AJ (2009) Neuroimaging in psychiatry: from bench to bedside. Front. Hum. Neurosci. 3:49. doi: 10.3389/neuro.09.049.2009 Copyright $\odot 2009$ Linden and Fallgatter. This is an open-access article subject to an exclusive license agreement between the authors and the Frontiers Research Foundation, which permits unrestricted use, distribution, and reproduction in any medium, provided the original authors and source are credited. 